\title{
On the relaxed mean-field stochastic control problem *
}

\author{
Khaled Bahlali ${ }^{\dagger}$
}

Meriem Mezerdi $\ddagger$

\author{
Brahim Mezerdi ${ }^{\S}$
}

November 9, 2018

\begin{abstract}
This paper is concerned with optimal control problems for systems governed by mean-field stochastic differential equation, in which the control enters both the drift and the diffusion coefficient. We prove that the relaxed state process, associated with measure valued controls, is governed by an orthogonal martingale measure rather that a Brownian motion. In particular, we show by a counter example that replacing the drift and diffusion coefficient by their relaxed counterparts does not define a true relaxed control problem. We establish the existence of an optimal relaxed control, which can be approximated by a sequence of strict controls. Moreover under some convexity conditions, we show that the optimal control is realized by a strict control.
\end{abstract}

Key words: Mean-field stochastic differential equation; relaxed control; martingale measure; approximation; tightness; weak convergence.

MSC 2010 subject classifications, 93E20, 60H30.

\section{Introduction}

In this paper, we deal with optimal control of systems driven by mean-field stochastic differential equations (MFSDE) of the form

$$
\left\{\begin{array}{l}
d X_{t}=b\left(t, X_{t}, E\left(\Psi\left(X_{t}\right)\right), u_{t}\right) d t+\sigma\left(t, X_{t}, E\left(\Phi\left(X_{t}\right)\right), u_{t}\right) d W_{t} \\
X_{0}=x
\end{array}\right.
$$

MFSDEs are obtained as limits of some interacting particle systems. This kind of approximation result is called "propagation of chaos", which says that when the number of particles (players) tends to infinity, the equations defining the evolution of the particles could be replaced by a single equation, called the McKeanVlasov equation. This mean-field equation, represents in some sense the average behavior of the infinite number of particles, see [26, 16] for details. Since the earlier papers [17, [14, mean-field control theory has raised a lot of interest, motivated by applications to various fields such as game theory, mathematical finance, communications networks, management of oil ressources. The typical example is the continuoustime Markowitz's mean-variance portfolio selection model, where one should minimize an objective function involving a quadratic function of the expectation, due to the variance term. The main drawback, when dealing with such mean-field stochastic control problems, is that the Bellmann principle of optimality does not hold. For this kind of problems, the stochastic maximum principle, provides a powerful tool to solve them, see [1, 6, 7, 9, 10, 18, 27. One can refer also to the recent book [2] and the references therein.

Our main goal in this work is to investigate existence of optimal controls. As it is well known for classical control problems, in the absence of Fillipov convexity conditions, an optimal strict control may fail to exist. In this case, the set of strict controls should be embedded into a wider class of measure valued controls, called relaxed controls. This class enjoys good compactness and convexity properties. The problem now is to define precisely the MFSDE associated to a relaxed control. At first look, one is tempted as in [8], to replace simply the drift and diffusion coefficient by their relaxed couterparts ie: the integrals of the drift and diffusion coefficient with respect to the relaxed control, adopting the same method as in deterministic

\footnotetext{
* Partially supported by the French Algerian Cooperation Program, Tassili 13 MDU 887.

${ }^{\dagger}$ Laboratoire IMATH, Université du Sud-Toulon-Var, B.P 20132, 83957 La Garde Cedex 05, France. E-mail: bahlali@univtln.fr

$\ddagger$ Laboratory of Applied Mathematics, University of Biskra, Po. Box 145, Biskra (07000), Algeria. E-mail: m_mezerdi@yahoo.fr

$\S$ Laboratory of Applied Mathematics, University of Biskra, Po. Box 145, Biskra (07000), Algeria. E-mail: bmezerdi@yahoo.fr
} 
control. As it will be shown in a simple counter example, the suggested "relaxed" state equation is not continuous with respect to the control variable. This implies in particular that the value functions for the original and relaxed problems are not the same. In addition, there is no mean to prove the existence of an optimal control for this model. So that the proposed "relaxed" model in [8] is not a true extension of the original control problem. The fundamental reason is that one has to relax the quadratic variation, of the stochastic integral part of the state equation, which is a Lebesgue integral, rather than the stochastic integral itself. Roughly speaking, the idea is to relax the generator of the process, which is intimately linked to the weak solutions of the relaxed stochastic equation, rather than the equation itself. As it will be shown, the stochastic equation associated with the relaxed generator will be governed by a continuous orthogonal martingale measure, rather than a Brownian motion. For this model, we prove that the strict and relaxed control problems have the same value function and that an optimal relaxed control exists. Our result extends in particular [4, 3, 11, 21, 22, to mean field controls and [5] to the case of a MFSDE with a controlled diffusion coefficient. The proof is based on tightness properties of the underlying processes and Skorokhod selection theorem. Moreover, due to the compactness of the action space, we show that the relaxed control could be choosen among the so-called sliding controls, which are convex combinations of Dirac measures. As a consequence and under the so-called Fillipov convexity condition, the optimal relaxed control is shown to be strict.

\section{Existence of optimal relaxed controls}

\subsection{Controlled mean field stochastic differential equations}

Let $\left(W_{t}\right)$ is a $d$-dimensional Brownian motion, defined on a probability space $(\Omega, \mathcal{F}, P)$, endowed with a filtration $\left(\mathcal{F}_{t}\right)$, satisfying the usual conditions. Let $\mathbb{A}$ be some compact metric space called the control set.

We study the existence of optimal controls for systems driven non linear mean-field stochastic differential equations (MFSDE in short) of the form:

$$
\left\{\begin{array}{l}
d X_{t}=b\left(t, X_{t}, E\left(\Psi\left(X_{t}\right)\right), u_{t}\right) d t+\sigma\left(t, X_{t}, E\left(\Phi\left(X_{t}\right)\right), u_{t}\right) d W_{t} \\
X_{0}=x
\end{array}\right.
$$

The cost functional over the time interval $[0, T]$ is given by

$$
J(u)=E\left(\int_{0}^{T} h\left(t, X_{t}, E\left(\varphi\left(X_{t}\right)\right), u_{t}\right) d t+g\left(X_{T}, E\left(\lambda\left(X_{T}\right)\right)\right),\right.
$$

where $b, \sigma, l, h, g$ and $\psi$ are given functions. The control variable $u_{t}$ called a strict control, is a measurable, $\mathcal{F}_{t}$ - adapted process with values in the action space $\mathbb{A}$. We denote $\mathcal{U}_{a d}$ the space of strict controls. Let us point out that the probability space and Brownian motion may change with the control $u$.

The objective is to minimize the cost functional $J(u)$ over the space $\mathcal{U}_{a d}$ ie: find $u^{*} \in \mathcal{U}_{a d}$ such that $J\left(u^{*}\right)=\inf \left\{J(u) ; u \in \mathcal{U}_{a d}\right\}$.

The following assumptions will be in force throughout this paper.

$\left(\mathbf{H}_{1}\right)$ Assume that

$$
\begin{gathered}
b:[0, T] \times \mathbb{R}^{d} \times \mathbb{R}^{d} \times \mathbb{A} \longrightarrow \mathbb{R}^{d} \\
\sigma:[0, T] \times \mathbb{R}^{d} \times \mathbb{R}^{d} \times \mathbb{A} \longrightarrow \mathbb{R}^{d} \otimes \mathbb{R}^{d} \\
\Psi: \mathbb{R}^{d} \longrightarrow \mathbb{R}^{d}, \Phi: \mathbb{R}^{d} \longrightarrow \mathbb{R}^{d}
\end{gathered}
$$

are bounded continuous functions such that $b(t, ., ., a), \sigma(t, ., ., a), \Psi($.$) and \Phi($.$) are Lipschitz continuous,$ uniformly in $(t, a)$.

$\left(\mathbf{H}_{\mathbf{2}}\right)$ Assume that

$$
\begin{aligned}
& h:[0, T] \times \mathbb{R}^{d} \times \mathbb{R}^{d} \times \mathbb{A} \longrightarrow \mathbb{R} \\
& g: \mathbb{R}^{d} \times \mathbb{R}^{d} \longrightarrow \mathbb{R} \\
& \varphi: \mathbb{R}^{d} \longrightarrow \mathbb{R}^{d} \\
& \lambda: \mathbb{R}^{d} \longrightarrow \mathbb{R}^{d}
\end{aligned}
$$

are bounded continuous functions and $h(t, ., ., a)$ is Lipschiz continuous uniformly in $(t, a)$. 
Proposition 2.1. Under assumption $\left(\mathbf{H}_{\mathbf{1}}\right)$ the MFSDE (2.1) has a unique strong solution, such that for every $p>0$ we have $E\left(\left|X_{t}\right|^{p}\right)<+\infty$.

Proof.

Let us define $\bar{b}(t, x, \mu, a)$ and $\bar{\sigma}(t, x, \mu, a)$ on $[0, T] \times \mathbb{R}^{d} \times \mathbb{P}_{2}\left(\mathbb{R}^{d}\right) \times \mathbb{A}$ by

$\bar{b}(t, x, \mu, a)=b\left(t, x, \int \Psi(x) d \mu(x), a\right)$ and $\bar{\sigma}(t, x, \mu, a)=\sigma\left(t, x, \int \Phi(x) d \mu(x), a\right)$, where $\mathbb{P}_{2}\left(\mathbb{R}^{d}\right)$ denotes the space of probability measures in $\mathbb{R}^{d}$, having a finite second order moment.

According to [16] Prop.1.2, it is sufficient to check that $\bar{b}$ and $\bar{\sigma}$ are Lipschitz in $(x, \mu)$ where $\mathbb{P}_{2}\left(\mathbb{R}^{d}\right)$ is equipped with the 2 -Wasserstein metric $d(\mu, \nu)=\inf \left\{\left(E^{Q}|X-Y|^{2}\right)^{1 / 2} ; Q \in \mathbb{P}_{2}\left(\mathbb{R}^{d} \times \mathbb{R}^{d}\right)\right.$, with marginals $\left.\mu, \nu\right\}$.

This follows from the Lipschitz continuity of $b$ and $\sigma$ with respect to $(x, y)$.

Using similar techniques as in [16] Prop.1.2, it holds that for each $p>0, E\left(\left|X_{t}\right|^{p}\right)<+\infty$.

\section{Examples of mean-field control problems}

\section{1) Example 1: The mean-variance portfolio selection problem}

Consider a financial market, in which two securities are traded continuously. The first is a bond, with price $S_{t}^{0}$ at time $t \in[0, T]$ governed by

$$
d S_{t}^{0}=S_{t}^{0} \rho_{t} d t, S_{0}^{0}=s_{0}>0
$$

The second is a stock with unit price $S_{t}^{1}$ at time $t \in[0, T]$ governed by

$$
d S_{t}^{1}=S_{t-}^{1}\left(b_{t} d t+\sigma_{t} d B_{t}\right), S_{0}^{1}=s^{1}>0
$$

The coefficients $\rho_{t}>0, b_{t}, \sigma_{t}$ are deterministic and bounded functions. For an investor, a portfolio $\pi$ is a process representing the amount of money invested in the stock. The wealth process $x^{x_{0}, \pi}$ corresponding to initial capital $x_{0}>0$, and portfolio $\pi$, satisfies then the equation

$$
\left\{\begin{array}{l}
d x_{t}=\left(\rho_{t} x_{t}+\pi_{t}\left(b_{t}-\rho_{t}\right)\right) d t+\pi_{t} \sigma_{t} d B_{t}, \text { for } t \in[0, T] \\
x_{0}=x
\end{array}\right.
$$

The objective is to maximize the mean terminal wealth $\mathbb{E}\left[x_{T}^{\pi}\right]$, and at the same time, to minimize the variance of the terminal wealth $\operatorname{Var}\left[x_{T}^{\pi}\right]$, over controls $\pi$ valued in $\mathbb{R}$. Then, the mean-variance portfolio optimization problem is to minimize the cost $J$, given by

$$
J(\pi)=-\mathbb{E}\left[x_{T}\right]+\mu \operatorname{Var}\left[x_{T}\right],
$$

subject to (4.3), where $\mu>0$. The admissible portfolio is assumed to be progressively measurable square integrable process, and such that the corresponding $x_{t}^{\pi} \geq 0$, for all $t \in[0, T]$. We denote by $\Pi$ the class of such strategies.

Note that the cost functional (4.4) may be rewritten in men-field terms as

$$
J(\pi)=\mathbb{E}\left[-x_{T}+\mu\left(x_{T}+\mathbb{E}\left[-x_{T}\right]\right)^{2}\right] .
$$

2) Example 2 : Mean-field-type game with one risk-sensitive decision-maker

The mean-field state equation is given by

$$
\left\{\begin{array}{l}
d X_{t}=\bar{b}\left(t, X_{t}, P_{X_{t}}, u_{t}\right) d t+\sigma\left(t, X_{t}, P_{X_{t}}\right) d W_{t} \\
X_{0}=x
\end{array}\right.
$$

the drift term $\bar{b}$ has the special form $\bar{b}=\left(\int|b|^{\alpha}\left(., t, X_{t}, y, u(t)\right) P_{X_{t}}(t, d y)\right)^{\frac{1}{\alpha}}$

The risk-sensitive control problem is to minimize the following cost functional

$$
J^{\theta}(u(\cdot))=\frac{1}{\theta} \log E\left(\exp \theta\left[\int_{0}^{T} h\left(t, X_{t}, P_{X_{t}}, u_{t}\right) d t+g\left(X_{T}, P_{X_{T}}\right)\right]\right)
$$

This kind of MFSDE models has been used to understand muscle contraction. Other similar models have been widely studied in chemical kinetics, statistical mechanics and economics, [27] for further details. 


\subsection{The relaxed control problem}

As it is well known in control theory that in the absence of convexity conditions, an optimal control may fail to exist in the set $\mathcal{U}_{a d}$ of strict controls (see e.g. [13, 19, 28]). To be convinced let us consider the following examples.

\section{Example 1.}

Minimize $J(u)=\int_{0}^{1}(X(t))^{2} d t$ over the set $\mathcal{U}_{a d}$ of measurable functions $u:[0,1] \rightarrow\{-1,1\}$, where $X^{u}(t)$ is the solution of $d X(t)=u(t) d t, X(0)=0$. We have $\inf _{u \in \mathcal{U}_{a d}} J(u)=0$.

Indeed, consider the sequence of Rademacher functions:

$$
u_{n}(t)=(-1)^{k} \text { if } \frac{k}{n} \leq t \leq \frac{(k+1)}{n}, 0 \leq k \leq n-1
$$

Then clearly $\left|X^{u_{n}}(t)\right| \leq 1 / n$ and $\left|J\left(u_{n}\right)\right| \leq 1 / n^{2}$ which implies that $\inf _{u \in \mathcal{U}_{a d}} J(u)=0$. There is however no control $\widehat{u}$ such that $J(\widehat{u})=0$. If this were the case, then for every $t, X^{\widehat{u}}(t)=0$. This in turn would imply that $\widehat{u}_{t}=0$, which is impossible.

Example 2. Minimize $J(u)=E\left[\int_{0}^{T}\left[X^{u}(t)^{2}+(1-u(t))^{2}\right] d t\right]$ subject to $d X^{u}(t)=u(t) d t+d W$, $X^{u}(0)=0$, where $W$ is one dimensional Brownian motion, $u$ is an open-loop control that is a measurable function from $[0, T]$ into $[-1,1]$. The optimal control minimizes the functional $\int_{0}^{T}\left[\widehat{X}^{u}(t)^{2}+(1-u(t))^{2}\right] d t$ where $\widehat{X}^{u}(t)=E\left[X^{u}(t)\right]$.

It is not difficult to prove that the family of Rademacher functions is a minimizing sequence $\left(\lim J\left(u_{n}\right)=\right.$ 0 ), then it follows that $\inf _{u \in \mathcal{U}_{a d}} J(u)=0$. But there is no control $u \in \mathcal{U}_{a d}$ satisfying $J(u)=0$, since it would have to satisfy $\widehat{X}^{u}(t)=0$ and $|u(t)|=1$ a.e. at the same time, which is impossible.

The problem in both examples is that the sequence $\left(u_{n}\right)$ has no limit in the space of strict controls. This limit, if it exists, would be the natural candidate for optimality.

These examples suggest that the set of strict controls is too narrow and should be embedded into a wider class of relaxed controls, with nice compactness properties. For the relaxed model, to be a true extension of the original control problem, the following both conditions must be satisfied:

i) The value functions of the original and the relaxed control problems must be equal.

ii) The relaxed control problem must have an optimal solution.

The idea of relaxed control is to replace the $\mathbb{A}$-valued process $\left(u_{t}\right)$ with a $\mathbb{P}(\mathbb{A})$-valued process $\left(\mu_{t}\right)$, where $\mathbb{P}(\mathbb{A})$ is the space of probability measures equipped with the topology of weak convergence. Then $\left(\mu_{t}\right)$ may be identified as a random product measure on $[0, T] \times \mathbb{A}$, whose projection on $[0, T]$ coincides with Lebesgue measure.

Let $\mathbb{V}$ be the set of product measures $\mu$ on $[0, T] \times \mathbb{A}$ whose projection on $[0, T]$ coincides with the Lebesgue measure $d t$. It is clear that every $\mu$ in $\mathbb{V}$ may be disintegrated as $\mu=d t . \mu_{t}(d a)$, where $\mu_{t}(d a)$ is a transition probability. The elements of $\mathbb{V}$ are called Young measures in deterministic theory, see [28].

$\mathbb{V}$ as a closed subspace of the space of positive Radon measures $\mathbb{M}_{+}([0, T] \times \mathbb{A})$ is compact for the topology of weak convergence. In fact it can be proved that it is compact also for the topology of stable convergence, where test functions are measurable, bounded functions $f(t, a)$ continous in $a$, see [11, 15] for further details.

Definition 2.2. A relaxed control on the filtered probability space $\left(\Omega, \mathcal{F}, \mathcal{F}_{t}, P\right)$ is a random variable $\mu=$ $d t . \mu_{t}(d a)$ with values in $\mathbb{V}$, such that $\mu_{t}(d a)$ is progressively measurable with respect to $\left(\mathcal{F}_{t}\right)$ and such that for each $t, 1_{(0, t]} \cdot \mu$ is $\mathcal{F}_{t}$-measurable. Let us denote $\mathcal{R}$ the set of relaxed controls.

Remark 2.3. The set $\mathcal{U}_{a d}$ of strict controls is embedded into the set $\mathcal{R}$ of relaxed controls by identifying $u_{t}$ with $d t \delta_{u_{t}}(d a)$.

Let us come back to the first example. If we identify $u_{n}(t)$ with the Dirac measure $\delta_{u_{n}(t)}(d u)$, then it is not difficult to prove that the sequence of product measures $\left(d t \delta_{u_{n}(t)}(d u)\right)_{n}$ converges weakly to $(d t / 2) \cdot\left[\delta_{-1}+\right.$ $\left.\delta_{1}\right](d a)$.

Let us define the relaxed model by 


$$
x_{t}^{q}=x_{0}+\int_{0}^{t} d s \int_{U} u q(s, d a)
$$

and the associated relaxed cost is given by

$$
J(q)=\int_{0}^{1}\left(x_{t}^{q}\right)^{2} d t
$$

Then it is clear that the strict control problem is generalized by the relaxed problem, by taking measures $q$ of the form

$$
q(d t, d u)=\delta_{u_{t}}(d u) d t
$$

Moreover if

$$
\widehat{q}(d t, d u)=\frac{1}{2}\left[\delta_{-1}+\delta_{1}\right](d u) d t
$$

then we have $J(\widehat{q})=0$ and $\widehat{q}$ as an optimal relaxed control. Moreover since $\inf _{u \in \mathcal{U}_{a d}} J(u)=\inf _{q \in \mathcal{R}} J(q)=0$, then the value functions of the strict and relaxed control problems are the same.

\subsubsection{Discussion on the relaxation of the state process}

A natural question arises: what is the natural SDE associated with a relaxed control. Let us point out that in the deterministic case or in the stochastic case where only the drift is controlled, one has just to replace in equation (2.1) the drift by the same drift integrated against the relaxed control. Now we are in a situation where both the drift and diffusion coefficient are controlled. Let us try a direct relaxation of the original equation as in [8]. The "relaxed" control problem will be governed by the MFSDE

$$
\left\{\begin{array}{l}
d X_{t}=\int_{A} b\left(t, X_{t}, E\left(\Psi\left(X_{t}\right)\right), a\right) \mu_{t}(d a) d t+\int_{A} \sigma\left(t, X_{t}, E\left(\Psi\left(X_{t}\right)\right), a\right) \mu_{t}(d a) d W_{t} \\
X_{0}=x
\end{array}\right.
$$

As it will be shown in the sequel, this model does not fullfill the requirements of a true relaxed model. The reason is that the relaxed process is not continuous in the control variable and as a consequence, the value functions of the original and relaxed control problems are not equal. Let us consider the following example.

Consider the control problem governed by the following SDE without mean-field terms:

$$
\left\{\begin{array}{l}
d X_{t}=u_{t} d W_{t} \\
X_{0}=x
\end{array}\right.
$$

where the control $u \in \mathcal{U}_{a d}$ : the set of measurable functions $u:[0,1] \rightarrow \mathbb{A}=[-1,1]$.

The "relaxed" model will be governed by the equation

$$
\left\{\begin{array}{l}
d X_{t}=\int_{\mathbb{A}} a \mu_{t}(d a) d W_{t} \\
X_{0}=x
\end{array}\right.
$$

Consider the sequence of Rademacher functions

$$
u_{n}(t)=(-1)^{k} \text { if } \frac{k}{n} \leq t \leq \frac{(k+1)}{n}, 0 \leq k \leq n-1 .
$$

Lemma 2.4. Let $d t . \delta_{u_{n}(t)}(d a)$ the relaxed control associated to $u_{n}(t)$, then the sequence $\left(d t . \delta_{u_{n}(t)}(d a)\right)$ converges weakly to $d t(1 / 2)\left(\delta_{-1}+\delta_{1}\right)(d a)$.

Proof. See [19] Lemma 1.1, page 20 
Remark 2.5. The sequence of Rademacher functions is a typical example of a minimising sequence with no limit in the set of strict controls. However its weak limit $d t(1 / 2)\left(\delta_{-1}+\delta_{1}\right)(d a)$ is an optimal relaxed control [19, 28].

It is clear that $X_{t}^{n}=\int_{0}^{t} u_{n}(s) \cdot d W_{s}=\int_{0}^{t}\left[\int_{\mathbb{A}} a \delta_{u_{n}(s)}(d a)\right] d W_{s}$ is a continuous martingale with quadratic variation $\left\langle X^{n}, X^{n}\right\rangle_{t}=\int_{0}^{t} u_{n}^{2}(s) . d s=t$. Therefore $\left(X_{t}^{n}\right)$ is a Brownian motion.

By Lemma 2.4, the sequence of relaxed controls $\left(d t . \delta_{u_{n}(t)}(d a)\right)$ converges weakly in $\mathbb{M}_{+}([0, T] \times \mathbb{A})$ to $\mu^{*}=(1 / 2) d t\left(\delta_{-1}+\delta_{1}\right)(d a)$. Let $X^{*}$ be the relaxed state process corresponding to the limit $\mu^{*}$, then

$$
X^{*}(t)=\int_{0}^{t} \int_{\mathbb{A}} a \cdot(1 / 2)\left(\delta_{-1}+\delta_{1}\right)(d a) d W_{t}=0
$$

It is obvious that the sequence of state processes $\left(X_{t}^{n}\right)$ cannot converge in $L^{2}$ to $X_{t}^{*}$. Indeed

$$
E\left[\left|X_{t}^{n}-X_{t}^{*}\right|^{2}\right]=E\left[\left|X_{t}^{n}\right|^{2}\right]=E\left[\left|\int_{0}^{t} u_{n}(s) \cdot d W_{s}\right|^{2}\right]=\int_{0}^{t} u_{n}^{2}(s) \cdot d s=t
$$

This implies in particular that the state process is not continuous in the control variable and as a byproduct, the value functions of the strict and "relaxed" control problems are not equal. Moreover, even if the set $\mathbb{V}$ is compact, there is no mean to prove existence of an optimal control for this model.

\section{What is the right relaxed state process?}

The reason why the proposed model in [8] is not a true extension of the original strict control problem, is that the stochastic integral part does not behave as a Lebesgue integral. In fact, one should relax the drift and the quadratic variation of the martingale part, which is a Lebesgue integral.

In the relaxed model, the quadratic variation process must be $\int_{0}^{t} \int_{A} \sigma \sigma^{*}\left(t, X_{t}, E\left(\Phi\left(X_{t}\right)\right), a\right) \mu_{t}(d a) d t$, which is more natural than relaxing the stochastic integral itself. Now, one has to look for a square integrable martingale whose quadratic variation is given by $\int_{0}^{t} \int_{\mathbb{A}} \sigma \sigma^{*}\left(t, X_{t}, E\left(\Phi\left(X_{t}\right), a\right) \mu_{t}(d a) d t\right.$, which is equivalent to the search of an object which is a martingale whose quadratic variation is $d t \mu_{t}(d a)$. This object is precisely a continuous orthogonal martingale measure, whose covariance measure is $d t \mu_{t}(d a)$. This is equivalent to the relaxation of the infinitesimal generator associated to the state process.

Following [16] Prop. 1.10, existence of a weak solution of equation (2.1) is equivalent to existence of a solution for the non linear martingale problem:

$$
f\left(X_{t}\right)-f\left(X_{0}\right)-\int_{0}^{t} L^{P_{X_{s}}} f\left(s, X_{s}, u_{s}\right) d s \text { is a } P \text {-martingale, }
$$

for every $f \in C_{b}^{2}$, for each $t>0$, where $L$ is the infinitesimal generator associated with equation (2.1),

$$
L^{\nu} f(t, x, a)=\frac{1}{2} \sum_{i, j}\left(a_{i, j} \frac{\partial^{2} f}{\partial x_{i} \partial x_{j}}\right)(t, x, a)+\sum_{i}\left(b_{i} \frac{\partial f}{\partial x_{i}}\right)(t, x, a),
$$

$b=b(t, x,\langle\Psi, \nu\rangle, a)$ and $a_{i, j}=\sigma \sigma^{*}(t, x,\langle\Phi, \nu\rangle, a), \nu \in \mathbb{M}_{1}\left(\mathbb{R}^{d}\right)$.

The natural relaxed martingale problem associated to the relaxed generator is defined as follows:

$$
f\left(X_{t}\right)-f\left(X_{0}\right)-\int_{0}^{t} \int_{\mathbb{A}} L^{P_{X_{s}}} f\left(s, X_{s}, a\right) \mu_{s}(d a) d s \text { is a } P \text { - martingale }
$$


for each $f \in C_{b}^{2}$, for each $t>0$.

Now, what is the stochastic differential equation corresponding to the relaxed martingale problem (2.5)? The answer is given by the following theorem.

Theorem 2.6. 1)Let $P$ be the solution of the martingale problem (2.5). Then $P$ is the law of a $d-$ dimensional adapted and continuous process $X$ defined on an extension of the space $\left(\Omega, \mathcal{F}, \mathcal{F}_{t}, P\right)$ and solution of the following MFSDE starting at x:

$$
\left\{\begin{array}{l}
d X_{t}=\int_{\mathbb{A}} b\left(t, X_{t}, E\left(\Psi\left(X_{t}\right)\right) a\right) \mu_{t}(d a) d t+\int_{\mathbb{A}} \sigma\left(t, X_{t}, E\left(\Phi\left(X_{t}\right), a\right) M(d a, d t),\right. \\
X_{0}=x
\end{array}\right.
$$

where $M=\left(M^{k}\right)_{k=1}^{d}$ is a family of d-strongly orthogonal continuous martingale measures, each having intensity $d t \mu_{t}(d a)$.

2) If the coefficients $b$ and $\sigma$ are Lipschitz in $x$, $y$, uniformly in $t$ and a, the SDE (2.6) has a unique pathwise solution.

Proof. 1) The proof is based essentially on the same arguments as in [12 Theorem IV-2 and [16] Prop. 1.10 .

2) The coefficients being Lipschitz continuous, following the same steps as in [16] and [12, it is not difficult to prove that Equation (2.6) has a unique solution such that for every $p>0$ we have $E\left(\left|X_{t}\right|^{p}\right)<+\infty$.

Remark 2.7. Note that the othogonal martingale measure corresponding to the relaxed control $d t \mu_{t}(d a)$ is not unique.

Let us give the precise definition of a martingale measure introduced by Walsh [29], see also [12, 20] for more details.

Definition 2.8. Let $\left(\Omega, \mathcal{F}, \mathcal{F}_{t}, P\right)$ be a filtered probability space, and $M(t, B)$ a random process, where $B \in \mathcal{B}(\mathbb{A}) . M$ is a $\left(\mathcal{F}_{t}, P\right)$-martingale measure if:

1)For each $B \in \mathcal{B}(\mathbb{A}),(M(t, B))_{t>0}$ is a square integrable martingale, $M(0, B)=0$.

2)For every $t>0, M(t,$.$) is a \sigma$-finite $L^{2}$-valued measure.

It is called continuous if for each $B \in \mathcal{B}(\mathbb{A}), M(t, B)$ is continuous and orthogonal if $M(t, B) \cdot M(t, C)$ is a martingale whenever $B \cap C=\phi$.

Remark 2.9. When the martingale measure $M$ is orthogonal, it is proved in 29] the existence of a random positive $\sigma$-finite measure $\mu(d t, d a)$ on $[0, T] \times \mathbb{A}$ such that $\langle M(., B), M(., B)\rangle_{t}=\mu([0, t] \times B)$ for all $t>0$ and $B \in \mathcal{B}(\mathbb{A}) . \mu(d t, d a)$ is called the covariance measure of $M$.

Example Let $\mathbb{A}=\left\{a_{1}, a_{2}, \cdots, a_{n}\right\}$ be a finite set. Then every relaxed control $d t \mu_{t}(d a)$ will be a convex combination of the Dirac measures $d t \mu_{t}(d a)=\sum_{i=1}^{n} \alpha_{t}^{i} d t \delta_{a_{i}}(d a)$, where for each $i, \alpha_{t}^{i}$ is a real-valued progressively measurable process, such that $0 \leq \alpha_{t}^{i} \leq 1$ and $\sum_{i=1}^{n} \alpha_{t}^{i}=1$. It is obvious that the solution of the relaxed martingale problem 2.5 is the law of the solution of the SDE

$$
d X_{t}=\sum_{i=1}^{n} b\left(t, X_{t}, E\left(\Psi\left(X_{t}\right)\right), a_{i}\right) \alpha_{t}^{i} d t+\sum_{i=1}^{n} \sigma\left(t, X_{t}, E\left(\Psi\left(X_{t}\right)\right), a_{i}\right)\left(\alpha_{t}^{i}\right)^{1 / 2} d B_{t}^{i}, \quad X_{0}=x,
$$

where the $B^{i}$ s are independent $d$-dimensional Brownian motions, on an extension of the initial probability space. The process $M$ defined by

$$
M(A \times[0, t])=\sum_{i=1}^{n} \int_{0}^{t}\left(\alpha_{s}^{i}\right)^{1 / 2} 1_{\left\{a_{i} \in A\right\}} d B_{s}^{i}
$$

is in fact an orthogonal continuous martingale measure (cf. [11, 29]) with intensity $\mu_{t}(d a) d t=\sum \alpha_{t}^{i} \delta_{a_{i}}(d a) d t$. Thus, the SDE (2.7) can be expressed in terms of $M$ and $\mu$ as follows:

$$
d X_{t}=\int_{\mathbb{A}} b\left(t, X_{t}, E\left(\Psi\left(X_{t}\right)\right) a\right) \mu_{t}(d a) d t+\int_{\mathbb{A}} \sigma\left(t, X_{t}, E\left(\Phi\left(X_{t}\right)\right), a\right) M(d a, d t)
$$




\subsubsection{Approximation of the relaxed model}

The relaxed control problem is now driven by equation

$$
\left\{\begin{array}{l}
d X_{t}=\int_{\mathbb{A}} b\left(t, X_{t}, E\left(\Psi\left(X_{t}\right)\right) a\right) \mu_{t}(d a) d t+\int_{\mathbb{A}} \sigma\left(t, X_{t}, E\left(\Phi\left(X_{t}\right)\right), a\right) M(d a, d t), \\
X_{0}=x
\end{array}\right.
$$

and accordingly the relaxed cost functional is given by

$$
J(\mu)=E\left(\int_{0}^{T} \int_{\mathbb{A}} h\left(t, X_{t}, E\left(\varphi\left(X_{t}\right)\right), a\right) \mu_{t}(d a) d t+g\left(X_{T}, E\left(\lambda\left(X_{T}\right)\right)\right) .\right.
$$

We show in this section that the strict and the relaxed control problems have the same value function. This is based on the chattering lemma and the stability of the state process with respect to the control variable.

Lemma 2.10. (Chattering lemma) i) Let $\left(\mu_{t}\right)$ be a relaxed control. Then there exists a sequence of adapted processes $\left(u_{t}^{n}\right)$ with values in $\mathbb{A}$, such that the sequence of random measures $\left(\delta_{u_{t}^{n}}(d a) d t\right)$ converges in $\mathbb{V}$ to $\mu_{t}(d a) d t, P-a . s$.

ii) For any $g$ continuous in $[0, T] \times \mathbb{M}_{1}(\mathbb{A})$ such that $g(t,$.$) is linear, we have P-$ a.s

$$
\lim _{n \rightarrow+\infty} \int_{0}^{t} g\left(s, \delta_{u_{s}^{n}}\right) d s=\int_{0}^{t} g\left(s, \mu_{s}\right) d s \text { uniformly in } t \in[0, T] .
$$

Proof. See [11] and [13] Lemma 1 page 152.

Proposition 2.11. 1) Let $\mu=\mu_{t}(d a) d t$ a relaxed control. Then there exist a continuous orthogonal martingale measure $M(d t, d a)$ whose covariance measure is given by $\mu_{t}(d a) d t$.

2) If we denote $M^{n}(t, B)=\int_{0}^{t} \int_{B} \delta_{u_{s}^{n}}(d a) d W_{s}$, where $\left(u^{n}\right)$ is defined as in the last Lemma, then for every bounded predictable process $\varphi: \Omega \times[0, T] \times \mathbb{A} \rightarrow \mathbb{R}$, such that $\varphi(\omega, t,$.$) is continuous, we have$

$$
E\left[\left(\int_{0}^{t} \int_{\mathbb{A}} \varphi(\omega, t, a) M^{n}(d t, d a)-\int_{0}^{t} \int_{\mathbb{A}} \varphi(\omega, t, a) M(d t, d a)\right)^{2}\right] \rightarrow 0 \text { as } n \longrightarrow+\infty,
$$

for a suitable Brownian motion $W$ defined on an eventual extension of the probability space.

Proof. See [20] pages 196-197.

Proposition 2.12. 1) Let $X_{t}, X_{t}^{n}$ be the solutions of state equation (2.6) corresponding to $\mu$ and $u^{n}$, where $\mu$ and $u^{n}$ are defined as in the chattering lemma. Then

$$
\lim _{n \rightarrow \infty} E\left[\sup _{0 \leq t \leq T}\left|X_{t}^{n}-X_{t}\right|^{2}\right]=0 .
$$

2) Let $J\left(u^{n}\right)$ and $J(\mu)$ be the expected costs corresponding respectively to $u^{n}$ and $\mu$, then $\left(J\left(u^{n}\right)\right)$ converges to $J(\mu)$.

\section{Proof.}

1)Let $\mu$ a relaxed control and $\left(d t \delta_{u_{t}^{n}}(d a)\right)$ the sequence of atomic measures associated to the sequence of strict controls $\left(u^{n}\right)$, as in the last Lemma. Let $X_{t}, X_{t}^{n}$ the corresponding state processes. If we denote $M^{n}(t, B)=\int_{0}^{t} \int_{B} \delta_{u_{s}^{n}}(d a) d W_{s}$, then $X^{n}$ may be written in a relaxed form

$$
\left\{\begin{array}{l}
d X_{t}^{n}=\int_{\mathbb{A}} b\left(t, X_{t}^{n}, E\left(\Psi\left(X_{t}^{n}\right)\right), a\right) \delta_{u_{t}^{n}}(d a) d t+\int_{\mathbb{A}} \sigma\left(t, X_{t}, E\left(\Phi\left(X_{t}\right)\right), a\right) M^{n}(d t, d a) \\
X_{0}=x
\end{array}\right.
$$


We have

$$
\begin{aligned}
\left|X_{t}-X_{t}^{n}\right| & \leq\left|\int_{0}^{t} \int_{\mathbb{A}} b\left(s, X_{s}, E\left(\Psi\left(X_{s}\right)\right), u\right) \mu_{s}(d a) \cdot d s-\int_{0}^{t} \int_{\mathbb{A}} b\left(s, X_{s}^{n}, E\left(\Psi\left(X_{s}^{n}\right)\right), u\right) \delta_{u_{s}^{n}}(d a) d s\right| \\
& +\left|\int_{0}^{t} \int_{\mathbb{A}} \sigma\left(s, X_{s}, E\left(\Phi\left(X_{s}\right)\right), a\right) M(d s, d a)-\int_{0}^{t} \int_{\mathbb{A}} \sigma\left(s, X_{s}^{n}, E\left(\Phi\left(X_{s}^{n}\right)\right), a\right) M^{n}(d s, d a)\right| \\
& \leq\left|\int_{0}^{t} \int_{\mathbb{A}} b\left(s, X_{s}, E\left(\Psi\left(X_{s}\right)\right), u\right) \mu_{s}(d a) \cdot d s-\int_{0}^{t} \int_{\mathbb{A}} b\left(s, X_{s}, E\left(\Psi\left(X_{s}\right)\right), a\right) \delta_{u_{s}^{n}}(d a) d s\right| \\
& +\left|\int_{0}^{t} \int_{\mathbb{A}} b\left(s, X_{s}, E\left(\Psi\left(X_{s}\right)\right), u\right) \delta_{u_{s}^{n}}(d a) \cdot d s-\int_{0}^{t} \int_{\mathbb{A}} b\left(s, X_{s}^{n}, E\left(\Psi\left(X_{s}^{n}\right)\right), a\right) \delta_{u_{s}^{n}}(d a) d s\right| \\
& +\sup _{s \leq t}\left|\int_{0}^{s} \int_{\mathbb{A}} \sigma\left(v, X_{v}, E\left(\Phi\left(X_{v}\right)\right), a\right) M(d v, d a)-\int_{0}^{t} \int_{\mathbb{A}} \sigma\left(v, X_{v}, E\left(\Phi\left(X_{v}\right)\right), a\right) M^{n}(d v, d a)\right| \\
& +\sup _{s \leq t} \mid \int_{0}^{s} \int_{\mathbb{A}} \sigma\left(v, X_{v}, E\left(\Phi\left(X_{v}\right), a\right) M^{n}(d v, d a)-\int_{0}^{t} \int_{\mathbb{A}} \sigma\left(v, X_{v}^{n}, E\left(\Phi\left(X_{v}^{n}\right), a\right) M^{n}(d v, d a) \mid\right.\right.
\end{aligned}
$$

Then by using Burkholder-Davis-Gundy inequality for the martingale part and the fact that all the functions in equation (2.6) are Lipschitz continuous, it holds that

$$
E\left(\sup _{0 \leq t \leq T}\left|X_{t}-X_{t}^{n}\right|^{2}\right) \leq K\left[\int_{0}^{T} E\left(\sup _{0 \leq s \leq t}\left|X_{s}-X_{s}^{n}\right|^{2}\right) d t+\varepsilon_{n}\right]
$$

where $K$ is a nonnegative constant and

$$
\begin{aligned}
\varepsilon_{n}= & E\left(\sup _{0 \leq t \leq T}\left|\int_{0}^{t} \int_{\mathbb{A}} b\left(s, X_{s}, E\left(\Psi\left(X_{s}\right)\right), u\right) \mu_{s}(d a) d s-\int_{0}^{t} \int_{\mathbb{A}} b\left(s, X_{s}, E\left(\Psi\left(X_{s}\right)\right), a\right) \delta_{u_{s}^{n}}(d a) d s\right|^{2}\right) \\
& +E\left(\sup _{0 \leq t \leq T}\left|\int_{0}^{t} \int_{\mathbb{A}} \sigma\left(s, X_{s}, E\left(\Psi\left(X_{s}\right)\right), a\right) M(d s, d a)-\int_{0}^{t} \int_{\mathbb{A}} \sigma\left(s, X_{s}, E\left(\Psi\left(X_{s}\right)\right), a\right) M^{n}(d s, d a)\right|^{2}\right) .
\end{aligned}
$$

By using Lemma 2.10 and the Lebesgue dominated convergence theorem, it holds that $\lim _{n \rightarrow+\infty} \varepsilon_{n}=0$. We conclude by using Gronwall's Lemma.

2) Property 1) implies that the sequence $\left(X_{t}^{n}\right)$ converges to $X_{t}$ in probability uniformly in $t$, then we have

$$
\begin{aligned}
\left|J\left(u^{n}\right)-J(\mu)\right| & \leq E\left[\int_{0}^{T} \int_{\mathbb{A}}\left|h\left(t, X_{t}^{n}, E\left(\varphi\left(X_{t}^{n}\right)\right), a\right)-h\left(t, X_{t}, E\left(\varphi\left(X_{t}\right)\right), a\right)\right| \delta_{u_{t}^{n}}(d a) d t\right] \\
& +E\left[\mid\left[\int_{0}^{T} \int_{\mathbb{A}} h\left(t, X_{t}, E\left(\varphi\left(X_{t}\right)\right), a\right) \delta_{u_{t}^{n}}(d a) d t-\int_{0}^{T} \int_{\mathbb{A}} h\left(t, X_{t}, E\left(\varphi\left(X_{t}\right)\right), a\right) \mu_{t}(d a) d t\right]\right] \\
& +E\left[\mid g\left(X_{T}^{n}, E\left(\lambda\left(X_{T}^{n}\right)\right)-g\left(X_{T}, E\left(\lambda\left(X_{T}\right)\right) \mid\right]\right.\right.
\end{aligned}
$$

It follows from the continuity and boundness of the functions $h, g, \varphi$ and $\lambda$ with respect to $x$ and $y$, that the first and third terms in the right hand side converge to 0 . The second term in the right hand side tends to 0 by the weak convergence of the sequence $\mu^{n}$ to $\mu$, the continuity and the boundedness of $h$ in the variable $a$. We use the dominated convergence theorem to conclude.

Remark 2.13. As a consequence of the last proposition, it holds that the infimum among relaxed controls is equal to the infimum among strict controls, that is the relaxed model is a true extension of the original control problem.

\subsection{Existence of optimal relaxed controls}

The following theorem which is the main result of this section, extends [4, 11, 13, to systems driven by mean field SDEs with controlled diffusion coefficient. 
Theorem 2.14. Under assumptions $\left(\mathbf{H}_{\mathbf{1}}\right),\left(\mathbf{H}_{\mathbf{2}}\right)$, there exist an optimal relaxed control.

The proof is based on some auxiliary Lemmas and will be given later.

Let $\left(\mu^{n}\right)_{n \geq 0}$ be a minimizing sequence, that is $\lim _{n \rightarrow \infty} J\left(\mu^{n}\right)=\inf _{q \in \mathcal{R}} J(\mu)$ and let $X^{n}$ be the unique solution of (2.6), associated with $\mu^{n}$ and $M^{n}$ where $M^{n}$ is a continuous orthogonal martingale measure with intensity $\mu^{n}$. We will prove that the sequence $\left(\mu^{n}, M^{n}, X^{n}\right)$ is tight and then show that we can extract a subsequence, which converges in law to a process $(\widehat{\mu}, \widehat{M}, \widehat{X})$, which satisfies the same MFSDE. To finish the proof we show that the sequence of cost functionals $\left(J\left(\mu^{n}\right)\right)_{n}$ converges to $J(\widehat{\mu})$ which is equal to $\inf _{\mu \in \mathcal{R}} J(\mu)$ and then we conclude that $(\widehat{\mu}, \widehat{M}, \widehat{X})$ is optimal.

Lemma 2.15. The sequence of distributions of the relaxed controls $\left(\mu^{n}\right)_{n}$ is relatively compact in $\mathbb{V}$.

Proof. The relaxed controls $\mu^{n}$ are random variables with values in the space $\mathbb{V}$ which is compact. Then Prohorov's theorem yields that the family of distributions associated to $\left(\mu^{n}\right)_{n \geq 0}$ is tight then it is relatively compact.

Lemma 2.16. The family of martingale measures $\left(M^{n}\right)_{n>0}$ is tight in the space $C\left([0,1], \mathcal{S}^{\prime}\right)$ of continuous functions from $[0,1]$ into $\mathcal{S}^{\prime}$, the topological dual of the Schwartz space $\mathcal{S}$ of rapidly decreasing functions.

Proof. The martingale measures $M^{n}, n \geq 0$, can be considered as random variables with values in $C\left([0,1], \mathcal{S}^{\prime}\right)$. By [23], Therem 5.1 , it is sufficient to show that for every $\varphi$ in $\mathcal{S}$ the family $\left(M^{n}(\varphi), n \geq 0\right)$ is tight in $C\left([0, T], \mathbb{R}^{d}\right)$ where $M^{n}(\omega, t, \varphi)=\int_{\mathbb{A}} \varphi(a) M^{n}(\omega, t, d a)$. Let $p>1$ and $s<t$, by the BurkholderDavis-Gundy inequality we have

$$
\begin{aligned}
E\left(\left|M_{t}^{n}(\varphi)-M_{s}^{n}(\varphi)\right|^{2 p}\right) & \leq C_{p} E\left[\left(\int_{s}^{t} \int_{\mathbb{A}}|\varphi(a)|^{2} \mu_{t}^{n}(d a) d t\right)^{p}\right] \\
& \leq C_{p} \sup _{a \in \mathbb{A}}|\varphi(a)|^{2 p}|t-s|^{p}=K_{p}|t-s|^{p}
\end{aligned}
$$

where $K_{p}$ is a constant depending on $p$ and $\varphi$. Then, the Kolmogorov tightness criteria in $C\left([0, T], \mathbb{R}^{d}\right)$ is fulfilled and the sequence $\left(M^{n}(\varphi)\right)$ is tight.

Lemma 2.17. The sequence $\left(X^{n}\right)_{n \geq 0}$ is tight in the space $C\left([0, T], \mathbb{R}^{d}\right)$

Proof. Let $p>1$ and $s<t$. Using usual arguments from stochastic calculus and the boundness of the coefficients $b$ and $\sigma$, it is easy to show that

$$
E\left(\left|X_{t}^{n}-X_{s}^{n}\right|^{2 p}\right) \leq C_{p}|t-s|^{p}
$$

which yields the tightness of $\left(X_{t}^{n}, n \geq 0\right)$ in $C\left([0, T], \mathbb{R}^{d}\right)$

\section{Proof. of Theorem 2.14}

By using the Lemmas 2.15, 2.16 and 2.17 , it holds that the sequence of processes $\left(\mu^{n}, M^{n}, X^{n}\right)$ is tight on the space $\Gamma=\mathbb{V} \times C\left([0,1], \mathcal{S}^{\prime}\right) \times C\left([0, T], \mathbb{R}^{d}\right)$. Then by the Skorokhod representation theorem, there exists a probability space $(\widehat{\Omega}, \widehat{\mathcal{F}}, \widehat{P})$, a sequence $\widehat{\gamma}^{n}=\left(\widehat{\mu}^{n}, \widehat{M^{n}}, \widehat{X^{n}}\right)$ and $\widehat{\gamma}=(\widehat{\mu}, \widehat{M}, \widehat{X})$ defined on this space such that:

(i) for each $n \in \mathbb{N}$, $\operatorname{law}\left(\gamma^{n}\right)=\operatorname{law}\left(\widehat{\gamma}^{n}\right)$,

(ii) there exists a subsequence $\left(\widehat{\gamma}^{n_{k}}\right)$ of $\left(\widehat{\gamma}^{n}\right)$, still denoted by $\left(\widehat{\gamma}^{n}\right)$, which converges to $\widehat{\gamma}, \widehat{P}$-a.s. on the space $\Gamma$.

This means in particular that the sequence of relaxed controls $\left(\widehat{\mu}^{n}\right)$ converges in the weak topology to $\widehat{\mu}$, $\widehat{P}-$ a.s. and $\left(\widehat{M^{n}}, \widehat{X^{n}}\right)$ converges to $(\widehat{M}, \widehat{X}), \widehat{\mathbb{P}}-$ a.s. in $C\left([0,1], \mathcal{S}^{\prime}\right) \times C\left([0, T], \mathbb{R}^{d}\right)$.

According to property (i), we get

$$
\left\{\begin{array}{l}
\widehat{X_{t}^{n}}=x+\int_{0}^{t} \int_{\mathbb{A}} b\left(s, \widehat{X_{s}^{n}}, E\left(\Psi\left(\widehat{X_{s}^{n}}\right)\right), a\right) \widehat{\mu}_{s}^{n}(d a) d s+\int_{0}^{t} \int_{\mathbb{A}} \sigma\left(s, \widehat{X_{s}^{n}}, E\left(\Phi\left(\widehat{X_{s}^{n}}\right)\right), a\right) \widehat{M^{n}}(d s, d a), \\
\widehat{X_{0}^{n}}=x
\end{array}\right.
$$


Since the coefficients $b, \sigma, \Psi$ and $\Phi$ are Lipschitz continuous in $(x, y)$, then according to property (ii) and using similar arguments as in 25] page 32, it holds that

$\int_{0}^{t} \int_{\mathbb{A}} b\left(s, \widehat{X_{s}^{n}}, E\left(\Psi\left(\widehat{X_{s}^{n}}\right)\right), a\right) \widehat{\mu}_{s}^{n}(d a) d s$ converges in probability to $\int_{0}^{t} \int_{\mathbb{A}} b\left(s, \widehat{X_{s}}, E\left(\Psi\left(\widehat{X_{s}}\right)\right), a\right) \widehat{\mu}_{s}(d a) d s$

and

$\int_{0}^{t} \int_{\mathbb{A}} \sigma\left(s, \widehat{X_{s}^{n}}, E\left(\Phi\left(\widehat{X_{s}^{n}}\right)\right), a\right) \widehat{M^{n}}(d s, d a)$ converges in probability to $\int_{0}^{t} \int_{\mathbb{A}} \sigma\left(s, \widehat{X_{s}}, E\left(\Phi\left(\widehat{X_{s}}\right)\right), a\right) \widehat{M}(d s, d a)$.

$b$ and $\sigma$ are Lipschitz continuous, therefore $\widehat{X}$ is the unique solution of the MFSDE

$$
\left\{\begin{array}{l}
\widehat{X_{t}}=x+\int_{0}^{t} \int_{\mathbb{A}} b\left(s, \widehat{X_{s}}, E\left(\Psi\left(\widehat{X_{s}}\right)\right), a\right) \widehat{\mu}_{s}(d a) d s+\int_{0}^{t} \int_{\mathbb{A}} \sigma\left(s, \widehat{X_{s}}, E\left(\Phi\left(\widehat{X}_{s}\right)\right), a\right) \widehat{M}(d s, d a), \\
\widehat{X}_{0}=x
\end{array}\right.
$$

To finish the proof of Theorem 2.14, it remains to check that $\widehat{\mu}$ is an optimal control.

The functions $b$ and $\sigma$ are Lipschitz continuous, then according to the above properties (i)-(ii) we get

$$
\begin{aligned}
\inf _{\mu \in \mathcal{R}} J(\mu) & =\lim _{n \rightarrow \infty} J\left(\mu^{n}\right), \\
& =\lim _{n \rightarrow \infty} E\left[\int_{0}^{T} \int_{\mathbb{A}} h\left(t, X_{t}^{n}, E\left(\varphi\left(X_{t}^{n}\right)\right), a\right) \mu_{t}^{n}(d a) d t+g\left(X_{T}^{n}, E \lambda\left(X_{T}^{n}\right)\right)\right] \\
& =\lim _{n \rightarrow \infty} \widehat{E}\left[\int_{0}^{T} \int_{\mathbb{A}} h\left(t, \widehat{X_{t}^{n}}, E\left(\varphi\left(\widehat{X_{t}^{n}}\right)\right), a\right) \widehat{\mu}_{t}^{n}(d a) d t+g\left(\widehat{X_{T}^{n}}, E \lambda\left(\widehat{X_{T}^{n}}\right)\right)\right] \\
& =\widehat{E}\left[\int_{0}^{T} \int_{\mathbb{A}} h\left(t, \widehat{X_{t}}, E\left(\varphi\left(\widehat{X_{t}}\right)\right), a\right) \widehat{\mu}_{t}(d a) d t+g\left(\widehat{X_{T}}, E \lambda\left(\widehat{X_{T}}\right)\right)\right] .
\end{aligned}
$$

Hence $\widehat{\mu}$ is an optimal control.

Remark 2.18. The proof of the last Theorem is based on tightness and weak convergence techniques. Then it is possible to prove it by using the non linear martingale problem and following the same steps as in 11 without using the pathwise representation of the solution.

We prove that in the next proposition that we can restrict the investigation for an optimal relaxed control to the class of so-called sliding controls also known as chattering controls, having the form

$$
\mu_{t}=\sum_{i=1}^{p} \alpha_{i}(t) \delta_{u_{i}(t)}(d a), u_{i}(t) \in \mathbb{A}, \alpha_{i}(t) \geq 0 \text { and } \sum_{i=1}^{p} \alpha_{i}(t)=1
$$

where $\alpha_{i}(t)$ and $u_{i}(t)$ are adapted stochastic processes.

Proposition 2.19. Let $\mu$ be a relaxed control and $X$ the corresponding state process. Then one can choose a sliding control

$$
\nu_{t}=\sum_{i=1}^{p} \alpha_{i}(t) \delta_{u_{i}(t)}(d a), u_{i}(t) \in A, \alpha_{i}(t) \geq 0 \text { and } \sum_{i=1}^{p} \alpha_{i}(t)=1
$$

such that

1) $X$ is a solution of the controlled MFSDE

$$
\left\{\begin{aligned}
d X_{t} & =\sum_{i=1}^{p} \alpha_{i}(t) b\left(t, X_{t}, E\left(\Psi\left(X_{t}\right)\right), u_{i}(t)\right) d t+\sum_{i=1}^{p} \alpha_{i}(t)^{1 / 2} \sigma\left(t, X_{t}, E\left(\Phi\left(X_{t}\right)\right), u_{i}(t)\right) d W_{t}^{i} \\
X_{0} & =x
\end{aligned}\right.
$$

where $\left(W^{i}\right)$ are independant Brownian motions defined on an extension of the probability space. 2) $J(\mu)=J(\nu)$, where $J(\nu)$ is associated with $X$. 


\section{Proof.}

Let $\Lambda$ denote the $d+d^{2}+1$-dimensional simplex

$$
\Lambda=\left\{\lambda=\left(\lambda_{0}, \lambda_{1}, \ldots, \lambda_{d+d^{2}+1}\right) ; \lambda_{i} \geq 0 ; \quad \sum_{i=0}^{d+d^{2}+1} \lambda_{i}=1\right\}
$$

and $W$ the $\left(d+d^{2}+2\right)$-cartesian product of the set $\mathbb{A}$

$$
W=\left\{w=\left(u_{0}, u_{1}, \ldots, u_{d+d^{2}+1}\right) ; u_{i} \in \mathbb{A}\right\}
$$

Define the function

$$
g(t, \lambda, w)=\sum_{i=0}^{d+d^{2}+1} \lambda_{i} \widetilde{b}\left(t, X_{t}, E\left(\Psi\left(X_{t}\right)\right), u_{i}\right)-\int_{A} \widetilde{b}\left(t, X_{t}, E\left(\Psi\left(X_{t}\right)\right), a\right) \mu_{t}(d a)
$$

where $t \in[0, T], \lambda \in \Lambda, w \in W$ and $\widetilde{b}\left(t, X_{t}, E\left(\Psi\left(X_{t}\right)\right), u_{i}\right)=\left(\begin{array}{c}b\left(t, X_{t}, E\left(\Psi\left(X_{t}\right)\right), u_{i}\right) \\ \sigma \sigma^{*}\left(t, X_{t}, E\left(\Phi\left(X_{t}\right)\right), u_{i}\right) \\ h\left(t, x_{t}, E\left(\varphi\left(X_{t}\right)\right), u_{i}\right)\end{array}\right)$

Let $\widetilde{b}\left(t, X_{t}, E\left(\Psi\left(X_{t}\right)\right), u_{i}\right), i=0,1, \ldots, d+d^{2}+1$, be arbitrary points in $P\left(t, X_{t}\right)$ where

$P\left(t, X_{t}\right)=\left\{\left(b\left(t, X_{t}, E\left(\Psi\left(X_{t}\right)\right), a\right), \sigma \sigma^{*}\left(t, X_{t}, E\left(\Phi\left(X_{t}\right)\right), a\right), h\left(t, X_{t}, E\left(\Psi\left(X_{t}\right)\right), a\right)\right) ; a \in \mathbb{A}\right\} \subset \mathbb{R}^{d+d^{2}+1}$

Then the convex hull of this set is the collection of all points of the form

$$
\sum_{i=0}^{d+d^{2}+1} \lambda_{i} \widetilde{b}\left(t, X_{t}, E\left(\Psi\left(X_{t}\right)\right), u_{i}\right)
$$

If $\mu$ is a relaxed control, then $\int_{\mathbb{A}} \widetilde{b}\left(t, X_{t}, E\left(\Psi\left(X_{t}\right)\right), a\right) \mu_{t}(d a) \in \operatorname{Conv}\left(P\left(t, X_{t}\right)\right)$, the convex hull of $P\left(t, X_{t}\right)$. Therefore it follows from Carathéodory's Lemma (which says that the convex hull of a $d$-dimensional set $M$ coincides with the union of the convex hulls of $d+1$ points of $M)$, that for each $(\omega, t) \in \Omega \times[0, T]$ the equation $g(t, \lambda, \omega)=0$ admits at least one solution. Moreover the set

$$
\left\{(\omega, \lambda, w) \in \Omega \times \Lambda \times W: \sum_{i=0}^{d+d^{2}+1} \lambda_{i} \widetilde{b}\left(t, X_{t}, E\left(\Psi\left(X_{t}\right)\right), u_{i}\right)=\int_{\mathbb{A}} \widetilde{b}\left(t, x_{t}, E\left(\Psi\left(x_{t}\right)\right), a\right) \mu_{t}(d a)\right\}
$$

is measurable with respect to $\mathcal{F}_{t} \otimes \mathcal{B}\left(\mathbb{R}^{d+1}\right) \otimes \mathcal{B}\left(\mathbb{A}^{d+1}\right)$ with non empty $\omega$-sections for each $\omega$.

Hence by using a measurable selection theorem [11, there exist measurable $\mathcal{F}_{t}$-adapted processes $\lambda_{t}$ and $w_{t}$ with values, respectively in $\Lambda$ and $W$ such that:

$$
\int_{\mathbb{A}} \widetilde{b}\left(t, X_{t}, E\left(\Psi\left(X_{t}\right)\right), a\right) \mu_{t}(d u)=\sum_{i=0}^{d+d^{2}+1} \lambda_{i}(t) \widetilde{b}\left(t, X_{t}, u_{i}(t)\right)
$$

Then it is easy to verify that the process defined by its drift $\sum_{i=0}^{d+d^{2}+1} \lambda_{i}(t) b\left(t, X_{t}, E\left(\Psi\left(X_{t}\right)\right), u_{i}(t)\right)$ and its quadratic variation $\sum_{i=0}^{d+d^{2}+1} \lambda_{i}(t) \sigma \sigma^{*}\left(t, X_{t}, E\left(\Psi\left(X_{t}\right)\right), u_{i}(t)\right)$ is the solution of the MFSDE (2.18), defined possibly on an extension of the initial probability space because of the possible degeneracy of the matrix $\sigma \sigma^{*}$. 
Corollary 2.20. Assume that the set

$$
P\left(t, X_{t}\right)=\left\{\widetilde{b}\left(t, X_{t}, E\left(\Psi\left(X_{t}\right)\right), a\right) ; a \in \mathbb{A}\right\} \subset \mathbb{R}^{d+d^{2}+1}
$$

is convex, where $\widetilde{b}\left(t, X_{t}, E\left(\Psi\left(X_{t}\right)\right), a\right)=\left(b\left(t, X_{t}, E\left(\Psi\left(X_{t}\right)\right), a\right), \sigma \sigma^{*}\left(t, X_{t}, E\left(\Phi\left(X_{t}\right)\right), a\right), h\left(t, X_{t}, E\left(\Psi\left(X_{t}\right)\right), a\right)\right)$.

Then the relaxed optimal control is realized by a strict control.

\section{Proof.}

Using Proposition 2.19, it follows that for each relaxed control $\mu$ we have

$$
\int_{\mathbb{A}} \widetilde{b}\left(t, X_{t}, E\left(\Psi\left(X_{t}\right)\right), a\right) \mu_{t}(d a) \in \operatorname{Conv}\left(P\left(t, X_{t}\right)\right)
$$

Since $P\left(t, X_{t}\right)$ is convex then $\operatorname{Conv}\left(P\left(t, X_{t}\right)\right)=P\left(t, X_{t}\right)$. Then applying the same arguments as in Proposition 2.19, there exists a measurable $\mathcal{F}_{t}$-adapted process $u_{t}$ such that

$$
\int_{\mathbb{A}} \widetilde{b}\left(t, X_{t}, E\left(\Psi\left(X_{t}\right)\right), a\right) \mu_{t}(d u)=\widetilde{b}\left(t, X_{t}, u_{t}\right)
$$

which implies that $X_{t}$ is a solution of the MFSDE

$$
\left\{\begin{array}{l}
d X_{t}=b\left(t, X_{t}, E\left(\Psi\left(X_{t}\right)\right), u_{t}\right) d t+\sigma\left(t, X_{t}, E\left(\Phi\left(X_{t}\right)\right), u_{t}\right) d W_{t} \\
X_{0}=x
\end{array}\right.
$$

and $J(\mu)=J(u)$. This ends the proof.

\section{Conclusion}

We have proved the existence of an optimal relaxed control for mean-field stochastic control problems, where both the drift and diffusion coefficient are controlled. The natural stochastic equation corresponding to a relaxed control is a mean field stochastic equation driven by an orthogonal martingale measure. As we have proved in a counter-example, replacing the drift and the diffusion coefficient by their relaxed counterparts in the relaxed equation, as in [8], does not lead to a true extension of the original problem. In fact, the case where the diffusion coefficient is controlled is not a direct extension of the deterministic case and reflects the stochastic nature of the problem.

\section{References}

[1] D. Andersson and B. Djehiche, A maximum principle for SDEs of mean-field type. Appl. Math. and Optim. 63(3) (2010), 341-356.

[2] A. Bensoussan, J. Frehse, P. Yam, Mean-field games and mean-field type control theory, Springer briefs in mathematics, 2013.

[3] S. Bahlali, B. Djehiche, B. Mezerdi, The relaxed stochastic maximum principle in singular optimal control of diffusions. SIAM J. Control Optim., Vol. 46 (2007), no. 2, 427-444

[4] S. Bahlali, B. Djehiche, B. Mezerdi, Approximation and optimality necessary conditions in relaxed stochastic control problems. J. Appl. Math. Stoch. Anal., Vol. 2006, Article ID 72762, 1-23.

[5] K. Bahlali, M. Mezerdi, B. Mezerdi, Existence of optimal controls for systems governed by mean-field stochastic differential equations, Afrika Statistika, Vol. 9 (2014), No 1, 627-645.

[6] R. Buckdahn, B. Djehiche, J. Li, A general stochastic maximum principle for SDEs of mean-field type. Appl. Math. Optim. 64 (2011), no. 2, 197-216.

[7] R. Carmona, F. Delarue, Probabilistic analysis of mean-field games. SIAM J. Control Optim. 51 (2013), no. $4,2705-2734$. 
[8] A. Chala, The relaxed optimal control problem for mean-field SDEs systems and application, Automatica 50 (2014), 924-930.

[9] F. Chighoub, B. Mezerdi, A stochastic maximum principle in mean-field optimal control problems for jump diffusions. Arab J. Math. Sci. 19 (2013), no. 2, 223-241.

[10] R. Elliott, X. Li, Y. H. Ni, Discrete time mean-field stochastic linear-quadratic optimal control problems. Automatica 49 (2013), no. 11, 3222-3233.

[11] N. El Karoui, D. H. Nguyen, and M. Jeanblanc-Picqué, Compactification methods in the control of degenerate diffusions: existence of an optimal control, Stochastics, 20 (1987), No. 3, 169-219.

[12] N. El Karoui, S. Méléard, Martingale measures and stochastic calculus, Probab. Th. and Rel. Fields 84 (1990), no. 1, 83-101.

[13] W. H. Fleming, Generalized solutions in optimal stochastic control, Differential Games and Control theory II, Proceedings of 2nd Conference, Univ. of Rhode Island, Kingston, RI, 1976, Lect. Notes in Pure and Appl. Math., 30, Marcel Dekker, New York, (1977), 147-165.

[14] M. Huang, R.P. Malhamé, P.E. Caines, (2006). Large population stochastic dynamic games: closed-loop McKean-Vlasov systems and the Nash certainty equivalence principle. Comm. in Inf. and Systems, 6 (2006), No3, 221-252.

[15] J. Jacod, J. Mémin, Sur un type de convergence intermédiaire entre la convergence en loi et la convergence en probabilité, Seminaire de Proba., XV, Lect. Notes in Math., Vol. 850 (1981), Springer, Berlin, 529-546.

[16] B. Jourdain, S. Méléard, W. Woyczynski, Nonlinear SDEs driven by Lévy processes and related PDEs. Alea 4, 1-29 (2008).

[17] J.M. Lasry, P.L. Lions, Mean-field games. Japan. J. Math., 2 (2007) 229-260.

[18] J. Li, Stochastic maximum principle in the mean-field controls. Automatica, Vol. 48, No 2 (2012), 366-373.

[19] L. Mazliak, An introduction to probabilistic methods in stochastic control. Lectures at Pescara Univ., April 1996.

[20] S. Méléard, Representation and approximation of martingale measures. Stoch. Partial Diff. Equ. and Their Appl., Lect. Notes in Control and Inf. Sc., Vol. 176, 1992, 188-199.

[21] B. Mezerdi, S. Bahlali, Approximation in optimal control of diffusion processes. Random Oper. Stochastic Equations, Vol. 8 (2000), no. 4, 365-372.

[22] B. Mezerdi, S. Bahlali, Necessary conditions for optimality in relaxed stochastic control problems, Stochastics and Stoch. Reports 73 (2002), no. 3-4, 201-218.

[23] I.Mitoma, Tightness of probabilities on $C\left([0,1] ; \mathcal{S}^{\prime}\right)$ and $D\left([0,1] ; \mathcal{S}^{\prime}\right)$ The Annals of Probab. $11(1983)$, no. 4, 989-999.

[24] S. Peng, A general stochastic maximum principle for optimal control problems. SIAM J. Control Optim., Vol. 28 (1990), No 4, 966-979.

[25] A.V. Skorokhod, Studies in the theory of random processes. Adisson-Wesley (1965), originally published in Kiev (1961).

[26] A.S. Sznitman, Topics in propagation of chaos. In Ecole de Probabilités de Saint Flour, XIX-1989. Lecture Notes in Math. 1464, pp. 165-251. Springer, Berlin (1989).

[27] H. Tembine, Risk-sensitive mean-field-type games with Lp-norm drifts, Automatica 59 (2015), $224-237$.

[28] M. Valadier, A course on Young measures. Rendiconti dell'Istituto di Matematica dell'Università di Trieste $01 / 1994$. 
[29] J.B. Walsh, An introduction to stochastic P.D.E., Lect. notes in Math. 1180 (1986), 265-439

[30] J. Yong and X.Y. Zhou, Stochastic controls, Hamiltonian Systems and HJB Equations, Springer, New York, (1999). 